\title{
Developmental Learning in a Pain-Related System: Evidence for a Cross-Modality Mechanism
}

\author{
Alexandra Waldenström, Jonas Thelin, Erik Thimansson, Anders Levinsson, and Jens Schouenborg \\ Section of Neurophysiology, Department for Physiological Sciences, Lund University, S-221 84 Lund, Sweden
}

The nociceptive spinal reflex system performs highly precise sensorimotor transformations that require functionally specified synaptic strengths. The specification is gradually attained during early development and appears to be learning dependent. Here we determine the time course of this specification for heat-nociceptive tail withdrawal reflexes and analyze which types of primary afferents are important for the learning by applying various forms of noninvasive sensory deprivations. The percentage of erroneous heat-nociceptive tail withdrawal reflexes (i.e., movements directed toward the stimulation) decreased gradually from $64.1 \pm 2.5 \%$ (mean \pm SEM) to $<10 \%$ during postnatal days $10-21$. This improvement was completely blocked by anesthetizing the tail during the adaptation period, confirming that an experience-dependent mechanism is involved in the specification of synaptic strengths. However, the results show that the adaptation occurs to a significant extent despite local analgesia and protection of the tail from noxious input, provided that tactile sensitivity is preserved. Therefore, it appears that a nociceptive input is not necessary for the adaptation, and that input from tactile receptors can be used to guide the nociceptive synaptic organization during development. Sensory deprivation in the adult rat failed to affect the heat-nociceptive withdrawal reflex system, indicating that the adaptation has a "critical period" during early development. These findings provide a key to the puzzle of how pain-related systems can be functionally adapted through experience despite the rare occurrence of noxious input during early life.

Key words: pain; somatosensory; activity-dependent learning and memory; spinal cord; sensorimotor transformation; development

\section{Introduction}

The nociceptive withdrawal reflex system has been used extensively as a model for pain-related processing in the spinal cord, sensorimotor integration, and learning in vertebrates (Schouenborg, 2002). Previous findings in mammals indicate that this system has a detailed modular organization that is functionally adapted during development through experience-dependent mechanisms (Holmberg and Schouenborg, 1996a,b; Holmberg et al., 1997). In the adult rat, each module performs a highly specific and functionally adapted sensorimotor transformation related to the action of the output muscle(s). For hindlimb reflexes, the receptive field location and sensitivity distribution closely match the pattern of withdrawal efficiency of the output muscle in a standing-like position (Schouenborg and Kalliomäki, 1990; Schouenborg and Weng, 1994; Levinsson et al., 2002). Therefore, the strengths of numerous connections are adapted to the shape of the body and movement patterns caused by single muscles, thereby allowing accurate transformations to take place. During postnatal development, the strengths of erroneous connections are reduced, whereas those of the acceptable ones be-

Received March 25, 2003; revised June 27, 2003; accepted June 30, 2003.

This work was supported by the Swedish Medical Research Council 1013, the Knut and Alice Wallenberg Foundation, the Magn. Bergvall Foundation, and the Johan and Greta Kocks Foundation. We thank Drs. Per Andersen, Anders Björklund, Maria Fitzgerald, Martin Garwicz, Sten Grillner, and Per Petersson for constructive criticism on this manuscript and AstraZeneca (Södertälje, Sweden) for providing the vehicle salve.

Correspondence should be addressed to Alexandra Waldenström, Department for Physiological Sciences, Section of Neurophysiology, Biomedical Center F10, S-221 84 Lund, Sweden. E-mail: Alexandra.Waldenstrom@mphy.lu.se. Copyright $\odot 2003$ Society for Neuroscience $\quad 0270-6474 / 03 / 237719-\bullet \$ 15.00 / 0$ come proportional to the withdrawal efficiency of the muscle (Guy and Abbott, 1992; Holmberg and Schouenborg, 1996b; Bizzi et al., 2000). During this process, the nociceptive withdrawal reflex can adapt both to altered peripheral innervation (Holmberg and Schouenborg, 1996a) and to experimentally altered movement patterns caused by neonatal tendon transfer (Holmberg et al., 1997). In the latter type of experiments, an altered withdrawal movement pattern caused by transferring the tendons of single muscles at birth resulted in a selective change in weight distribution of the nociceptive reflex connections to the manipulated muscles. Thus, the connection strengths in the nociceptive withdrawal reflex modules appear to be functionally specified through learning mechanisms during development. However, noxious input is relatively rare during development, and nociceptors appear to have a high threshold from birth onward (Fitzgerald, 1987; Falcon et al., 1996). Consequently, it is not clear how a pain-related system could be functionally adapted through experience.

The aim of the present study was to clarify whether nociceptive input is used for nociceptive withdrawal reflex adaptation and, if not, which type of cutaneous inputs are responsible. For this end, the following experiments were performed: First, the normal developmental time course of tail nociceptive withdrawal reflex adaptation was determined. Nociceptive $\mathrm{C}$ fiber-evoked withdrawal reflexes were elicited by $\mathrm{CO}_{2}$ laser noxious heat stimulation of the tail (Holmberg and Schouenborg, 1996b). Second, sensory deprivations targeting various combinations of tactile and/or nociceptive receptors were applied to the tail during the 
Table 1. Summary of treatments and resulting sensory deprivations

\begin{tabular}{|c|c|c|c|c|c|}
\hline Treatments & Noxious input & Nociceptive sensitivity & Tactile input & Tactile sensitivity & Number of rats \\
\hline Control & + & + & + & + & 22 \\
\hline Tube & - & + & + & + & 12 \\
\hline Tube and EMLA & - & - & + & + & 12 \\
\hline Tube and vehicle & - & + & + & + & 12 \\
\hline Tube and EMLA and depilation & - & - & $(+)$ & - & 10 \\
\hline Tube and vehicle and depilation & - & + & $(+)$ & $(+)$ & 10 \\
\hline
\end{tabular}

+ , Presence of input or sensitivity; - , absence of input or sensitivity; parentheses indicate absence of hair follicle input or sensitivity.

normal adaptation time period. Third, control experiments were conducted to evaluate whether the local application of analgesic salve to the tail has a systemic analgesic effect on the CNS and whether the sensory deprivation abolishes the adaptation mechanism. Finally, we investigated whether the heat-nociceptive withdrawal reflex adaptation is limited primarily to early development or whether a continuous sensory input is necessary to maintain adequate reflex performance in the adult.

\section{Materials and Methods}

Subjects. A total of 161 Wistar rats of both sexes, obtained from rats with known mating time, were included. Postnatal day 1 (P1) was defined as the first $24 \mathrm{hr}$ after birth. Skin temperature [measured with an infrared noncontact probe (Thermonitor C-1600 M; Linear Laboratories, Los Altos, CA)] was assessed daily on the tail, the back of the rat, and in some experiments, the nose. For animals used to determine the normal developmental time course of the nociceptive withdrawal reflex, body weight was measured daily up to P21 and thereafter every second day until P27. For animals included in the deprivation studies, body weight was measured just before and after the deprivation periods and the following observation days. Treated animals did not differ from controls with regard to these parameters. The general behavior was observed daily to ensure that the deprivation treatment did not cause inadvertent effects. Before treatment, the ears of all animals were labeled. The rats received food and water ad libitum and were kept in a $12 \mathrm{hr}$ light/dark cycle at a constant temperature of $21^{\circ} \mathrm{C}$ (humidity, $65 \%$ ). The Local Ethical Committee of the University of Lund approved all procedures.

Behavioral tests. The time course of the nociceptive withdrawal reflex adaptation and effects of various forms of sensory deprivations were determined with the same behavioral tests. Withdrawal reflexes were elicited bilaterally on the tail by a $\mathrm{CO}_{2}$ laser (unfocused beam: diameter, $1.1 \mathrm{~mm}$; intensity, $1 \mathrm{~W}$; pulse duration, $8-30 \mathrm{msec}$ ). To determine the reflex threshold $\left(T_{\mathrm{r}}\right)$ on each individual animal, the pulse duration was increased in 2 msec steps until three of five stimulations elicited responses. The reflex threshold was typically in the range of 8-12 msec. According to Haimi-Cohen et al. (1983), the threshold stimulations in rats (usually $12 \mathrm{msec}$ duration) will result in a temperature of $\sim 50^{\circ} \mathrm{C}$ at a skin depth of $100 \mu \mathrm{m}$. Reflex responses were elicited only at intensities that were painful when tested on the skin of the experimenters, supporting that heat nociceptors were activated at threshold stimulation intensity. In animals treated with analgesics (see below), the reflex threshold determined before the treatment (i.e., at P14), was used, because nociceptive reflex thresholds changed only marginally during the deprivation period in animals not treated with analgesics. To evoke reliable heatnociceptive withdrawal reflexes (Devor et al., 1982; Bromm and Treede, 1984), a stimulus intensity of $2 T_{\mathrm{r}}$ was used. To minimize "wind-up" in the nociceptive pathways (Mendell, 1966; Schouenborg, 1984; Woolf, 1996), the interval between stimulation of the same skin site was at least 10 min. Calibrated nylon von Frey monofilaments (North Coast Medical, San Jose, CA) with increasing stiffness (up to $90 \mathrm{mN}$ ) were used to determine the mechanical threshold (response in three of five trials) on the dorsal side of the tail.

Sensory deprivations and testing procedures. The animals used were put under brief general volatile anesthesia ( $2 \%$ halothane or $3-4 \%$ isoflurane, in a mixture of $70 \%$ nitrous oxide and $30 \%$ oxygen). Control animals received the same general anesthesia and were separated from their mothers for the same time periods as the treated animals. Animals in-
A
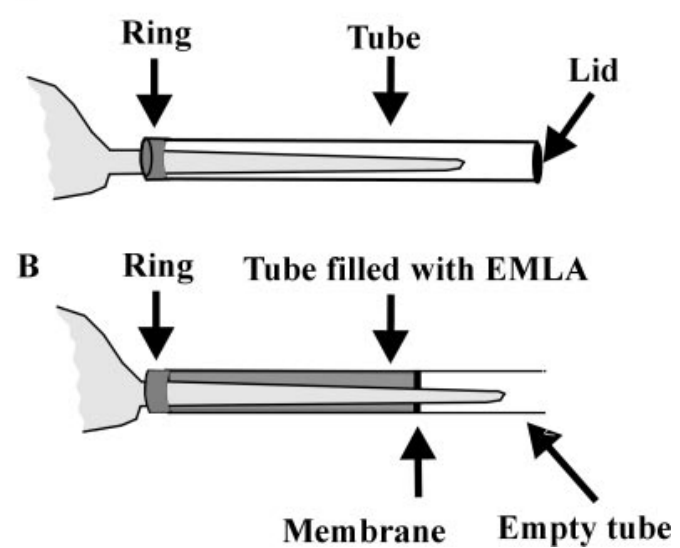

Figure 1. Method of sensory deprivation of the tail. $A$, Sensory deprivation of the tail was achieved by attaching a tube to a plastic ring glued to the ventral side of the proximal tail. The tube was either empty or filled with salve. $B$, A modified tube with two compartments separated by a membrane was used to study possible systemic effects of EMLA. Note that the plastic ring was glued more proximal on the tail than in $A$. The proximal compartment was filled with EMLA.

cluded in each treatment group were randomly picked from several litters. All of the different treatment groups are summarized in Table 1. Five different treatments were administered and compared with controls. Control animals received the same general anesthesia and were separated from their mothers for the same time periods as the treated animals. Group 1 was treated with a tube only. For this purpose, a plastic ring was glued (methylacrylate; Loctite 454; ELFA, Järfälla, Sweden) to the ventral side of the proximal tail. A hard plastic cylindrical tube (adapted to age; length, 50-100 mm; inner and outer diameter, 7 and $10 \mathrm{~mm}$; weight, 2.5-5 gm) with smooth inner walls was attached to the ring (Fig. $1 \mathrm{~A}$ ). This prevented noxious but not tactile input. Nociceptive and tactile sensitivity were not abolished. Group 2 was treated with a tube containing the analgesic salve EMLA (eutectic mixture of 2.5\% Prilocaine and 2.5\% Lidocaine for long-term analgesia; AstraZeneca, Södertälje, Sweden) (Buckley and Benfield, 1993). The tube was sealed with a lid (Fig. $1 A$ ), preventing noxious but not tactile input. Nociceptive but not tactile sensitivity was abolished. Group 3 was treated with a tube containing vehicle (salve without any analgesic compounds, provided by AstraZeneca), preventing noxious input. Neither tactile input nor nociceptive and tactile sensitivity were abolished. Group 4 was treated with a tube containing EMLA and was also depilated at P14. Depilation (i.e., removal of the hairs) of the tail was performed by removing a compress attached to the hairs with methylacrylate. Any remaining hairs were shaved. During the treatment period, additional depilations were performed when necessary. This treatment prevented noxious and hair follicle input and also abolished tactile and nociceptive sensitivity. Group 5 was treated with a tube containing vehicle and was also depilated at P14, which prevented noxious input and hair follicle input. Nociceptive and tactile sensitivity other than hair follicle sensitivity were not abolished.

Three more treatment groups were prepared for control purposes and to allow additional analysis of the adaptation mechanisms. One group was treated in the same way as group 4, but the depilation and EMLA treatments were applied during P17-P24 (i.e., when the error rate of the withdrawal reflex was adult-like). A second group was treated at P14- 


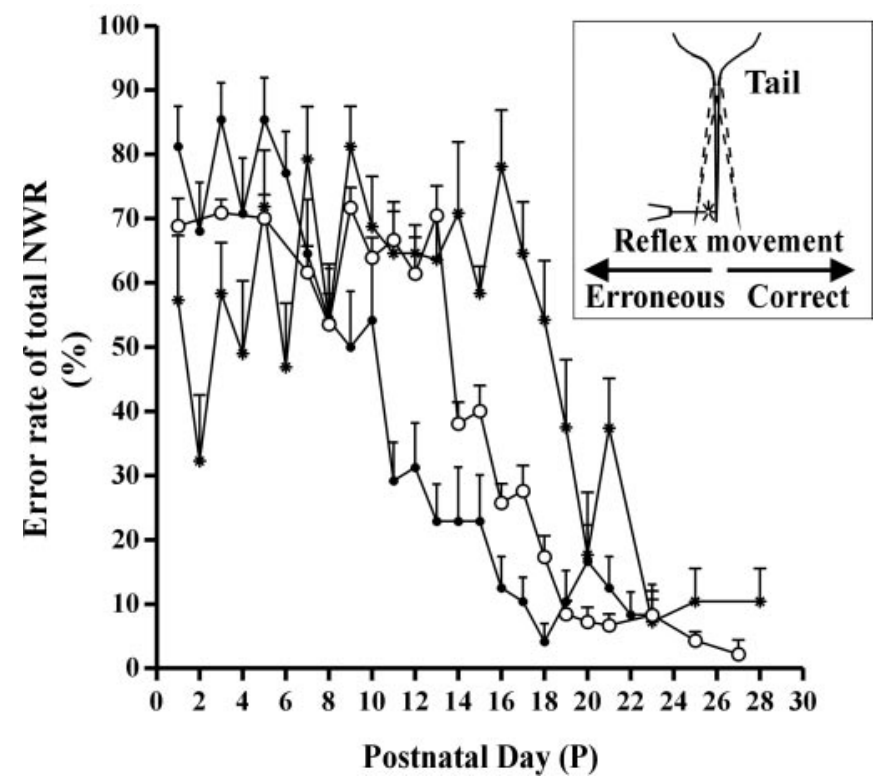

Figure 2. Time course of nociceptive withdrawal reflex (NWR) postnatal adaptation. Graph shows percentage of error rate of heat-nociceptive withdrawal reflex in three litters. The distal tail was stimulated daily, twice bilaterally during P1-P28 in two litters $\left(O n=12 ;{ }^{*} n=8\right)$, and six times on each side in a third litter $(\bigcirc n=13)$. Mean \pm SEM are shown. The inset shows a schematic of the experimental set-up. Tail movements in the horizontal plane, elicited by $\mathrm{CO}_{2}$ laser, were classified as erroneous (directed toward stimulation) or correct (directed away from stimulation).

P21 with a different tube construction consisting of two compartments. The proximal compartment was filled with EMLA and covered the proximal three-quarters of the tail (i.e., approximately the same surface area as in other EMLA-treated rats). The corresponding part of the tail was depilated at P14. A membrane between the two compartments prevented the distal part of the tail from being exposed to the EMLA salve. The distal compartment was empty, and the tail was not depilated (Fig. $1 B$ ). This treatment was used to study possible systemic effects of EMLA. A third group was treated for a week in the same way as groups 2 and 4 but in 2-month-old rats. In this case, an aluminum ring and tube (weight, $7 \mathrm{gm}$ ) was used for salve application. All tube constructions with or without salve allowed small tail movements inside the tube and near-normal flexibility at the proximal part of the tail. Animals showing any sign of disturbed tail circulation, such as edema, were excluded from the study $(n=6)$. This occurred when the applied glue used to attach the ring unintentionally covered more than the ventral side of the tail and interrupted circulation.

Behavioral tests were performed before and after sensory deprivation treatment. During the treatment period, the analgesic-anesthetic effect was checked every 12 th $\mathrm{hr}$ in the awake animal using noxious thermal $\mathrm{CO}_{2}$ laser stimulation (each animal was stimulated once on both sides of the tail at an intensity of $2 T_{\mathrm{r}}$ ) and tactile stimulation (as described above). After sensory testings, the tube was refilled with fresh EMLA or vehicle. After treatment, behavioral tests were performed when sensitivity had recovered (3-6 hr after removal of salves) and up to $5 \mathrm{~d}$ thereafter.

Analysis and statistics. The withdrawal reflex responses were recorded on videotape ( 50 frames/sec) and analyzed frame by frame by visual inspection. The analyses were performed blindly [i.e., the treatments used on the animals (see below) were not revealed to the analysts]. The heat-nociceptive withdrawal reflexes were analyzed and characterized with respect to the direction of the withdrawal movement [i.e., erroneous (directed toward stimulation) or correct (directed away from stimulation) (Fig. 2)]. The nonparametric $\chi^{2}$ test was used for statistical comparisons between all treated groups. Corrections for multiple comparisons were made using the Bonferroni method. Significant differences were assumed at a level of $p<0.05$. Values are given as mean \pm SEM.

\section{Results}

Time course for nociceptive withdrawal reflex adaptation

To identify a suitable period for somatosensory deprivation, we first studied the normal heat-nociceptive withdrawal reflex development from P1 up to P28. The nociceptive withdrawal reflexes were elicited daily by $\mathrm{CO}_{2}$ laser stimulation (intensity, $2 T_{\mathrm{r}}$ ) twice bilaterally on the distal tail during P1-P28 in two litters $(n=12$ and 8 , respectively) and six times on each side in a third litter $(n=$ 13). $\mathrm{CO}_{2}$ laser stimulation is known to selectively activate heatnociceptive fibers (Devor et al., 1982; Bromm and Treede, 1984). The daily noxious stimulations used did not affect the normal time course of the nociceptive withdrawal reflex adaptation [comparison between rats that had been stimulated daily $(n=$ $33)$ and rats that had not been stimulated before P14 $(n=78)$ and P17 $(n=11)$ ] (Holmberg and Schouenborg, 1996a).

Up to approximately P10, $75 \%$ of the movements were erroneously directed toward the noxious thermal input $(64.1 \pm$ $2.5 \%$ error rate at $\mathrm{P} 10$ ). By $\mathrm{P} 21$, the error rate in most rats was reduced to adult-like levels (i.e., <10\%) (Fig. 2). Note, however, that the adaptation time course differs between litters by several days. As a result, the error rate differed between all litters significantly during the interval P17-P18 $(p<0.05)$.

\section{Effects of sensory deprivation}

Based on the time course data (Fig. 2), rats with an error rate in the range of $45-55 \%$ at P14 were used for various forms of sensory deprivation during the entire third postnatal week (P14P21) (Table 1). The effect of these deprivations on the heatnociceptive withdrawal reflex adaptation was studied when sensitivity had recovered (3-6 hr after cessation of treatment). All groups were compared with one another.

\section{Effects of nociceptive input}

In one group of rats, the tail was protected by a hard plastic tube (Fig. 1A) to prevent noxious input. These rats showed no statistically significant difference in the error rate of heat-nociceptive withdrawal reflexes at P21 when compared with untreated animals of the same litter (Fig. 3, tube vs control). Thus, the protection against noxious input did not appear to have a marked effect on the heat-nociceptive withdrawal reflex adaptation. Moreover, it can be inferred that the tube itself and the consequent restriction of tail movements had no significant effect on the adaptation.

It is known that a small group of the cutaneous nociceptive afferents can be weakly activated by non-noxious mechanical pressure. In the rat, the lowest mechanical thresholds of nociceptors have been reported to be in the non-noxious range (Leem et al., 1993). Therefore, the possibility that such fibers can be weakly activated despite the protection of the tail with a tube cannot be excluded. To further assess the contribution of the low-threshold nociceptors for the adaptation of the heat-nociceptive withdrawal reflexes, tubes filled with the analgesic salve EMLA (Leem et al., 1993; Gazarian et al., 1995; Riou et al., 2000) were applied to one group of rats. It is known that EMLA blocks both mechanical and thermal nociception in humans, thus allowing skin surgery (Buckley and Benfield, 1993). This treatment caused local analgesia in the tail, which was tested twice daily using noxious $\mathrm{CO}_{2}$ laser stimulation (see Materials and Methods). The error rate of the heat-nociceptive withdrawal reflex decreased significantly $(p<0.01 ; \mathrm{P} 21$ compared with P14) in these rats. Thus, the heatnociceptive withdrawal reflexes adapt significantly despite analgesia and protection against noxious input by the tube. More- 


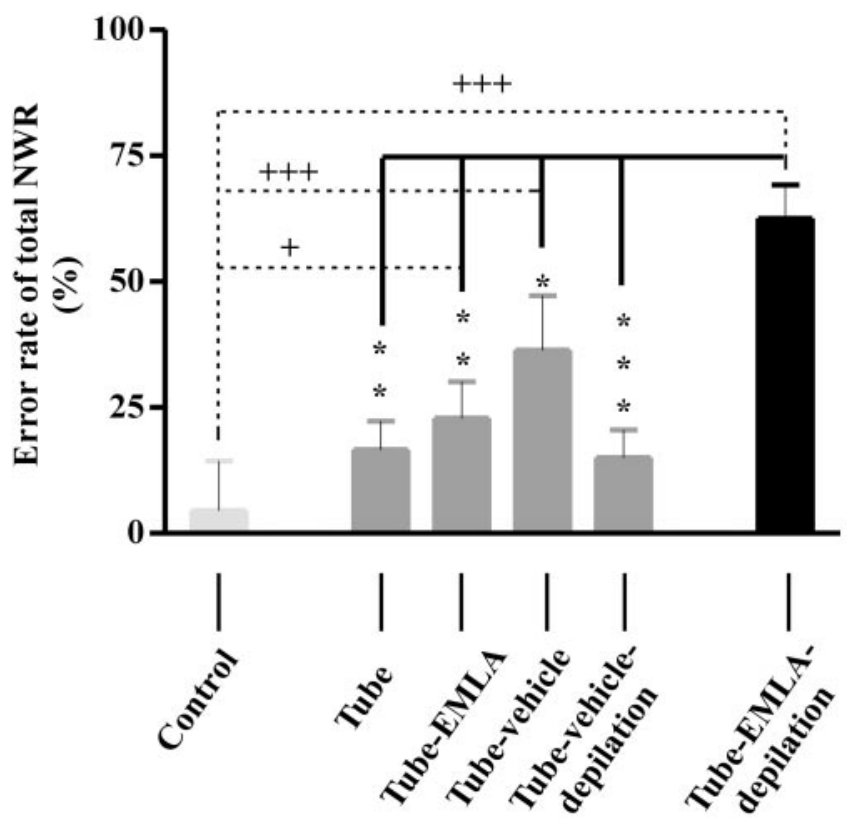

Figure 3. Effects of different forms of sensory deprivations applied during P14-P21 on the heat-nociceptive withdrawal reflex adaptation. Comparisons were made between all groups at P21. All significant differences are indicated. The gray bars signify treatment with deprived or absent nociceptive input but preserved tactile sensitivity. The black bar signifies treatment that produced anesthesia. Solid lines indicate comparisons with the group treated with tube-EMLA and depilation; ${ }^{*} p<0.05 ;{ }^{* *} p<0.01 ;{ }^{* * *} p<0.001$. Dotted lines indicate comparisons with the control group; ${ }^{+} p<0.05 ;{ }^{+++} p<0.001$. The $\chi^{2}$ test was used for statistics. Corrections for multiple comparisons were made using the Bonferroni method. NWR, Nociceptive withdrawal reflex.

over, the error rate after EMLA treatment did not differ significantly from that in animals treated with tubes filled with vehicle (Fig. 3, tube-EMLA and tube-vehicle). This finding indicates that the adaptation that occurred during these two treatments was attributable to an input from afferent fibers other than the cutaneous nociceptors. However, rats treated with EMLA or vehicle did not adapt as well as controls (Fig. 3, tube-EMLA and tube-vehicle vs control), possibly because of the mechanical dampening and blurring effect of the salves.

\section{Effects of tactile input}

The EMLA salve treatment did not reduce tactile sensitivity; this was assessed daily using calibrated nylon von Frey monofilaments (threshold at P14, $14.6 \pm 5.5 \mathrm{mN}$; threshold during the treatment period, $10.5 \pm 2.0 \mathrm{mN}$ ). Differential stimulation showed that both hair follicle afferents and other low-threshold mechanoreceptors were responsible for the tactile sensitivity. To assess the role of tactile input for the adaptation of the heatnociceptive withdrawal reflexes, we combined the tube and EMLA treatments with depilation (i.e., removal of the hairs) (see Materials and Methods) in one group of rats. This resulted in total cutaneous mechanical and nociceptive insensitivity in the tail. By P21, the heat-nociceptive withdrawal reflexes had not improved compared with the controls at P14 (Fig. 3). This was in sharp contrast to the rats with preserved tactile sensitivity (Fig. 3, tube-EMLA depilation vs tube, tube-EMLA, tube-vehicle, tubevehicle depilation: $p<0.01 ; p<0.01 ; p<0.05 ; p<0.01$, respectively), indicating a role for low-threshold mechanoreceptive afferents in the adaptation of heat-nociceptive withdrawal reflexes (also see control experiments below).

To further study the role of hair follicle receptors for the ad-
A

B

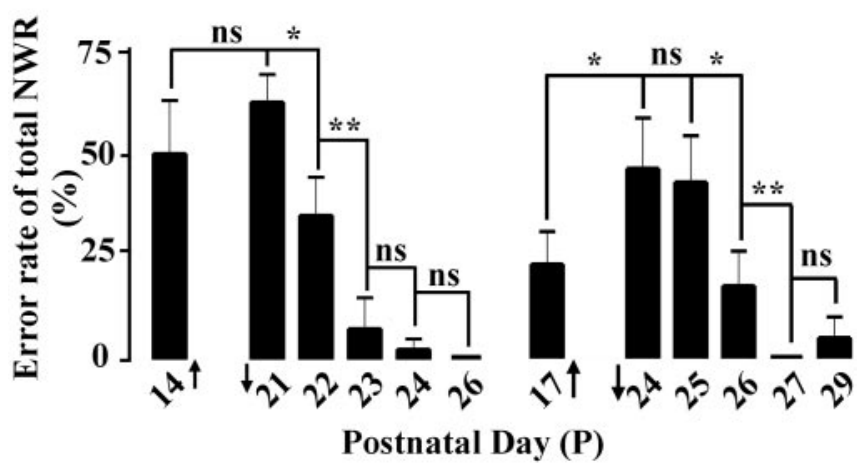

Figure 4. Time course of nociceptive withdrawal reflex (NWR) adaptation after complete sensory block. Tails were depilated and EMLA-treated in A (P14-P21) and B (P17-P24). Arrows indicate start and end of treatment ( $\uparrow$ and $\downarrow$, respectively). The error rate of heatnociceptive withdrawal reflex before and up to $5 \mathrm{~d}$ after treatment is shown. ${ }^{*} p<0.05$; ${ }^{* *} p<$ 0.01 ; ns, not significant. The $\chi^{2}$ test was used for statistics.

aptation, we also treated one group of rats with tube, vehicle, and depilation (Fig. 3, tube-vehicle depilation). These rats still responded to tactile stimulation during the treatment but were protected from noxious input by the tube and vehicle. The error rate in these rats decreased to a near-normal level at P21, indicating that hair follicle receptors are not necessary for the heatnociceptive withdrawal reflex adaptation.

\section{Reversal of adaptation}

To investigate whether the adaptation process is reversible during the normal adaptation period, animals that had developed a nearadult heat-nociceptive withdrawal reflex error rate were treated with EMLA and depilated during P17-P24 (Fig. $4 B)(n=11)$. This resulted in a significantly increased error rate $(p<0.05)$, indicating that much or all of the preceding adaptation was erased by the imposed lack of sensory input. These results suggest that synaptic weights in the reflex network are not stabilized during the normal adaptation period. After the treatment time, the error rate was reduced to an adult level within 2 or $3 \mathrm{~d}$ (Fig. $4 B$ ). This was also true for rats that were subjected to sensory deprivation at P14-P21 (Fig. 4A, tube-EMLA depilation). These findings indicate that the adaptation mechanisms are preserved after a period during which there has been no sensory input.

\section{Control experiments}

To exclude the possibility that the reduced heat-nociceptive withdrawal reflex adaptation after EMLA and depilation treatment compared with treatment with EMLA was attributable to systemic analgesic effects or to direct effects on the CNS (Woolf and Wiesenfeld-Hallin, 1985; Koppert et al., 2000), a similar area but more proximal part of the tail was anesthetized with EMLA and depilation in six animals during P14-P21. For this purpose, a modified tube with two compartments was used (see Materials and Methods) (Fig. $1 B$ ). In these animals, the error rate for the heat-nociceptive withdrawal reflex elicited from the distal tail decreased ( $p<0.001$; P21 compared with P14) to a level (29 \pm $4.2 \%)$ that was not significantly different from animals that had been treated with a tube only $(17 \pm 5.6 \%)$ or treated with EMLA only $(23 \pm 7.2 \%)$. Furthermore, the heat-nociceptive thresholds on the distal tail $(10.8 \pm 05 \mathrm{msec})$, hindpaw (14.0 $\pm 0.5 \mathrm{msec})$, and nose $(12.8 \pm 0.5 \mathrm{msec})$ did not differ significantly from the controls (distal tail, $12.4 \pm 0.4 \mathrm{msec}$; hindpaw, $14.3 \pm 0.4 \mathrm{msec}$; 
nose, $13.4 \pm 0.5 \mathrm{msec} ;=7$ ) during the treatment. These data and the lack of difference between vehicle- and EMLA-treated rats indicate that the abolished adaptation of the heat-nociceptive withdrawal reflexes after treatment with tube, EMLA, and depilation is not attributable to a general analgesic effect or to direct central effects of the EMLA salve on the adapting mechanisms.

\section{Effect of sensory deprivation in the adult}

To determine whether the heat-nociceptive withdrawal reflex adaptation is limited primarily to early development or whether a continuous sensory input is necessary to maintain adequate reflex performance in the adult, tails of adult rats ( 2 months of age) were continuously treated for 1 week with tube, EMLA, and depilation $(n=7)$; tube and EMLA $(n=8)$; and depilation only $(n=$ $7)$. In none of these groups did the error rate change significantly compared with control values before treatment. These data suggest that the heat-nociceptive withdrawal reflex network is relatively stable in the adult, and that sensory input is not required to sustain adequate withdrawal reflex performance.

\section{Discussion}

The present study provides a time table for nociceptive withdrawal reflex adaptation and confirms that it is dependent on sensory experience (see Introduction). It also provides evidence that this sensory experience can be mediated by tactile input, which represents a novel cross-modality learning principle (cf. Shimojo and Shams, 2001).

The data indicate that there is a critical time window for the nociceptive withdrawal reflex adaptation because the heatnociceptive withdrawal reflex performs adequately in young adult rats (2 months of age) but not in P14-P21 rats after a week of sensory deprivation. The demonstrated variability in the adaptation time course suggests that the end points of this time window can differ between litters by several days. In addition, it is likely that withdrawal reflexes of different body parts, although presumably organized in a similar way (Holmberg and Schouenborg, 1996a), exhibit somewhat different adaptation time courses, because there is a rostrocaudal difference in maturation of the spinal cord (Altman and Bayer, 1984; Holmberg and Schouenborg, 1996a).

Sensory systems such as the vibrissal, visual, and auditory systems require sensory input during a critical time window to be functionally adapted (Simons and Land, 1987; Katz, 1999; Knudsen et al., 2000). However, these systems require input from their own modality (Knudsen and Knudsen, 1986; Crair et al., 1998). For the nociceptive systems, noxious input is scarce during early development. Considering the highly detailed information that is required to functionally specify the weight of the numerous nociceptive afferent connections to withdrawal reflex modules (Schouenborg and Weng, 1994), it appears unlikely that nociceptive information is used for the nociceptive withdrawal reflex adaptation. Two main observations in the present study support this assumption: (1) protection of the tail from noxious input by a hard plastic tube failed to significantly affect the heatnociceptive withdrawal reflex adaptation, and (2) treatment of analgesics did not abolish the adaptation of the heat-nociceptive withdrawal reflexes and did not yield an effect on nociceptive withdrawal reflex adaptation different from that obtained by treatment with vehicle (Fig. 3, gray bars). This assumption is consistent with the observation that the daily noxious stimulations used did not affect the normal time course of the nociceptive withdrawal reflex adaptation (Holmberg and Schouenborg, 1996a). This does not mean that nociceptive input cannot affect the sensitivity in the nociceptive pathways (Woolf, 1996). For example, long-lasting increases in general pain sensitivity after intense noxious stimulation in the neonate have been reported previously (Taddio et al., 1997; Ruda et al., 2000; Iyengar and Bottjer, 2002).

It should be noted that no other types of cutaneous receptors besides nociceptive or tactile receptors contribute to the nociceptive withdrawal reflexes, and only these receptor types would be able to provide information about withdrawal efficacy (Weng and Schouenborg, 1998). Proprioceptors do not provide adequate information about withdrawal of the skin surface, and the different treatments with salves used here would not be expected to affect proprioceptive input differently. Thus, if nociceptors do not play a role in the adaptation of the heat-nociceptive withdrawal reflex, then low-threshold mechanoreceptors of the skin would seem to be the only remaining candidates. Importantly, the heat-nociceptive withdrawal reflex was found to adapt significantly if only tactile sensitivity is preserved (tube-EMLA-treated rats) but not in rats with blocked tactile and nociceptive sensitivity (treatment combining EMLA with depilation). These findings thus indicate that tactile input contributes to the adaptation of the heat-nociceptive withdrawal reflexes. Given that this assumption is correct, which mechanoreceptors are likely to contribute? Although hair follicle receptors and other low-threshold mechanoreceptors mediate the tactile sensitivity in rats treated with tube, tube-vehicle, or tube-EMLA, rats treated with tube-vehicle depilation are deprived of hair follicle input. The lack of a statistically significant difference between the four treatment groups may therefore suggest that hair follicle afferents are not necessary for the heat-nociceptive withdrawal reflex adaptation, at least not if other low-threshold mechanoreceptors are available to convey the tactile information. However, our findings do not exclude a role for hair follicle afferents in the adaptation. In fact, depilation tended (although not to a statistically significant extent) to cause an improved adaptation in the vehicle-treated animals (Fig. 3, tube-vehicle vs tube-vehicle depilation). Thus, hair follicle afferent input may have a detrimental effect on the adaptation in this situation. It should be noted that the tendency for less accurate adaptation in rats treated with tube, tube-EMLA, tube-vehicle, or tube-vehicle depilation compared with controls (Fig. 3 ) is the expected result of an experience-dependent mechanism. The tube and salve treatment has a mechanical dampening and blurring effect that inevitably reduces the precision of the tactile experience and thereby the accuracy of an experience-dependent mechanism.

Our findings provide evidence that a previously unknown cross-modality learning mechanism, which uses tactile information, underlies the adaptation of the nociceptive withdrawal reflex connections. This cross-modality learning is presumably greatly facilitated by the congruent topographical organization of the nociceptive and tactile termination in laminas II and III-IV of the dorsal horn, respectively (Schouenborg, 1984; Coggeshall et al., 1996; Levinsson et al., 2002). Indeed, the topography of the tactile input to laminas III-IV appears to be related to the topography of the nociceptive input to lamina V "reflex encoder" (RE) neurons in the lower lumbar cord (Levinsson et al., 2002). More specifically, the strength of the tactile input from different skin areas to a given region in the dorsal horn has a spatial pattern that mimics the sensitivity distribution within the receptive field of the underlying reflex encoders in lamina V. Moreover, the neurons in lamina II of the dorsal horn, some of which are likely to be first-order reflex interneurons, receive convergent tactile and nociceptive input from overlapping skin areas (Cervero and Iggo, 


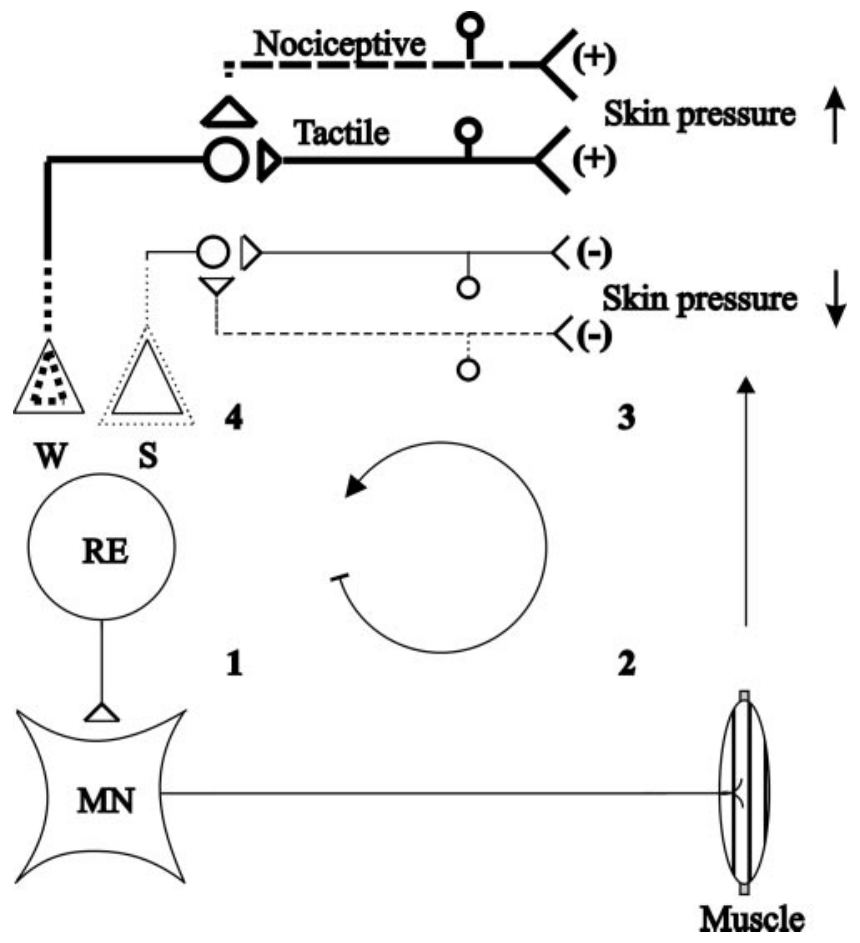

Figure 5. A proposed self-organizing circuitry that uses tactile information related to withdrawal efficacy to adjust the strength of nociceptive connections. One learning cycle (indicated by circular arrow) consists of the following chain of events: (1) spontaneous bursts in REs (i.e., neurons that encode the reflex magnitude); (2) motoneuron (MN) activation leading to a muscle twitch that causes skin movement (indicated by a thin long upward arrow); (3) increased or decreased skin pressure (indicated by upward and downward thick arrows, respectively) resulting in altered sensory input to prereflex encoder interneurons. Thick and thin lines represent afferents from skin areas on the tail from where an increase $(+)$ and decrease $(-)$, respectively, in low-threshold mechanoreceptor input would occur. Dashed lines symbolize the rare occurrence of nociceptive input; and (4) the strength of erroneous connections (receiving increased mechanoreceptive input) between pre-RE interneurons and RE is weakened (W) and that of appropriate ones (receiving reduced mechanoreceptive input) is strengthened (S). Dotted lines indicate that the number of pre-RE interneurons is not known. Evidence that synaptic strength can be reduced if action potentials in postsynaptic neurons precede activity in the presynaptic neuron has been presented for other systems (Debanne et al., 1994; Markram et al., 1997; Zhang and Po0, 2001). Note that although the nociceptive input is not required for the learning to take place in this model, nociceptive input, if present, would indeed cause learning effects.

1980). Given that tactile information about withdrawal efficiency is conveyed to each reflex module, a learning process could be conceived. The tactile input could be generated by the skin movements that ensue on muscle contractions (Schouenborg and Weng, 1994). The dominating behavior during early postnatal development consists of spontaneous muscle twitches (Narayanan et al., 1971; Wenner and O'Donovan, 2001) caused by spontaneous activity in the spinal cord (Visser et al., 1985; Blumberg and Lucas, 1994).

Based on these considerations, a learning mechanism in which tactile feedback after muscle twitches provides the necessary sensory information for nociceptive withdrawal reflex adaptation is tentatively proposed in Figure 5. The key features of this model are that: (1) the tactile and nociceptive input from the same skin area converge on prereflex encoder interneurons; (2) the strength of connections between pre-RE interneurons and RE interneurons is modifiable; and (3) twitches in unilateral muscles are caused by spontaneous activity in reflex encoder neurons. Indeed, evidence that tactile feedback after spontaneous muscle twitches modifies the nociceptive withdrawal reflexes has been obtained recently in our laboratory (Petersson et al., 2002). A "learning cycle" would thus be initiated by spontaneous activity in the reflex encoders, which, via muscle twitches, would cause tactile feedback related to the function of the withdrawal reflex circuitry (Fig. 5). In view of the protracted length of the nociceptive withdrawal reflex adaptation (Fig. 2; >1 week), each learning cycle would provide a small contribution to the change in synaptic strength between first-order interneurons and reflex encoders. The fact that a total block of sensory input could reverse the adaptation process suggests that the synaptic strength is at least temporarily labile and can be either increased or decreased.

In conclusion, the present findings provide a key to the paradox of how the pain system can be adapted through experience despite the rare occurrence of noxious input by demonstrating that tactile inputs can be used to adapt nociceptive connections in the nociceptive withdrawal reflex system. In addition, a selforganizing learning mechanism, which may explain how sensorimotor transformation in spinal reflex systems is functionally adapted during development, is proposed.

\section{References}

Altman J, Bayer SA (1984) The development of the rat spinal cord. Advances in anatomy embryology and cell biology, Vol 85. Berlin, Heidelberg, New York, Tokyo: Springer-Verlag.

Bizzi E, Tresch MC, Saltiel P, d'Avella A (2000) New perspectives on spinal motor systems. Nat Rev Neurosci 1:101-108.

Blumberg MS, Lucas DE (1994) Dual mechanisms of twitching during sleep in neonatal rats. Behav Neurosci 108:1196-1202.

Bromm B, Treede RD (1984) Nerve fibre discharges, cerebral potentials and sensations induced by $\mathrm{CO}_{2}$ laser stimulation. Hum Neurobiol 3:33-40.

Buckley MM, Benfield P (1993) Eutectic lidocaine/prilocaine cream. A review of the topical anaesthetic/analgesic efficacy of a eutectic mixture of local anaesthetics (EMLA). Drugs 46:126-151.

Cervero F, Iggo A (1980) The substantia gelatinosa of the spinal cord: a critical review. Brain 103:717-772.

Coggeshall RE, Jennings EA, Fitzgerald M (1996) Evidence that large myelinated primary afferent fibers make synaptic contacts in lamina II of neonatal rats. Brain Res Dev Brain Res 92:81-90.

Crair MC, Gillespie DC, Stryker MP (1998) The role of visual experience in the development of columns in cat visual cortex. Science 279:566-570.

Debanne D, Gahwiler BH, Thompson SM (1994) Asynchronous pre- and postsynaptic activity induces associative long-term depression in area CA1 of the rat hippocampus in vitro. Proc Natl Acad Sci USA 91:1148-1152.

Devor M, Carmon A, Frostig R (1982) Primary afferent and spinal sensory neurons that respond to brief pulses of intense infrared laser radiation: a preliminary survey in rats. Exp Neurol 76:483-494.

Falcon M, Guendellman D, Stolberg A, Frenk H, Urca G (1996) Development of thermal nociception in rats. Pain 67:203-208.

Fitzgerald M (1987) Cutaneous primary afferent properties in the hind limb of the neonatal rat. J Physiol (Lond) 383:79-92.

Gazarian M, Taddio A, Klein J, Kent G, Koren G (1995) Penile absorption of EMLA cream in piglets: implications for use of EMLA in neonatal circumcision. Biol Neonate 68:334-341.

Guy ER, Abbott FV (1992) The behavioral response to formalin in preweanling rats. Pain 51:81-90.

Haimi-Cohen R, Cohen A, Carmon A (1983) A model for the temperature distribution in skin noxiously stimulated by a brief pulse of $\mathrm{CO}_{2}$ laser radiation. J Neurosci Methods 8:127-137.

Holmberg H, Schouenborg J (1996a) Postnatal development of the nociceptive withdrawal reflexes in the rat: a behavioural and electromyographic study. J Physiol (Lond) 493:239-252.

Holmberg H, Schouenborg J (1996b) Developmental adaptation of withdrawal reflexes to early alteration of peripheral innervation in the rat. J Physiol (Lond) 495:399-409.

Holmberg H, Schouenborg J, Yu YB, Weng HR (1997) Developmental adaptation of rat nociceptive withdrawal reflexes after neonatal tendon transfer. J Neurosci 17:2071-2078.

Iyengar S, Bottjer SW (2002) The role of auditory experience in the forma- 
tion of neural circuits underlying vocal learning in zebra finches. J Neurosci 22:946-958.

Katz LC (1999) What's critical for the critical period in visual cortex? Cell 99:673-676.

Knudsen EI, Knudsen PF (1986) The sensitive period for auditory localization in barn owls is limited by age, not by experience. J Neurosci 6:1918-1924.

Knudsen EI, Zheng W, DeBello WM (2000) Traces of learning in the auditory localization pathway. Proc Natl Acad Sci USA 97:11815-11820.

Koppert W, Ostermeier N, Sittl R, Weidner C, Schmelz M (2000) Low-dose lidocaine reduces secondary hyperalgesia by a central mode of action. Pain 85:217-224.

Leem JW, Willis WD, Weller SC, Chung JM (1993) Differential activation and classification of cutaneous afferents in the rat. J Neurophysiol 70:2411-2424.

Levinsson A, Holmberg H, Broman J, Zhang M, Schouenborg J (2002) Spinal sensorimotor transformation: relation between cutaneous somatotopy and a reflex network. J Neurosci 22:8170-8182.

Markram H, Lubke J, Frotscher M, Sakmann B (1997) Regulation of synaptic efficacy by coincidence of postsynaptic APs and EPSPs. Science 275:213-215.

Mendell LM (1966) Physiological properties of unmyelinated fiber projection to the spinal cord. Exp Neurol 16:316-332.

Narayanan CH, Fox MW, Hamburger V (1971) Prenatal development of spontaneous and evoked activity in the rat (Rattus norvegicus albinus). Behaviour 40:100-134.

Petersson P, Waldenström A, Fåhreus C, Schouenborg J (2002) The postnatal tuning of the nociceptive withdrawal reflex is dependent on spontaneous motor activity: unsupervised learning in a spinal neuronal circuit, p 137. San Diego: 10th World Conference International Association for the Study of Pain.

Riou Y, Storme L, Klosowski S, Rakza T, Kongolo G, Zerimech F, MartinPonthieu A, Lequien P (2000) Combined effects of inhaled nitric oxide (iNO) and oxidant agents on the production of methemoglobinemia in newborn piglets. Crit Care Med 28:1068-1071.

Ruda MA, Ling QD, Hohmann AG, Peng YB, Tachibana T (2000) Altered nociceptive neuronal circuits after neonatal peripheral inflammation. Science 289:628-631.

Schouenborg J (1984) Functional and topographical properties of field potentials evoked in rat dorsal horn by cutaneous C-fibre stimulation. J Physiol (Lond) 356:169-192.

Schouenborg J (2002) Modular organisation and spinal somatosensory imprinting. Brain Res Brain Res Rev 40:80-91.

Schouenborg J, Kalliomäki J (1990) Functional organization of the nociceptive withdrawal reflexes. I. Activation of hindlimb muscles in the rat. Exp Brain Res 83:67-78.

Schouenborg J, Weng HR (1994) Sensorimotor transformation in a spinal motor system. Exp Brain Res 100:170-174.

Shimojo S, Shams L (2001) Sensory modalities are not separate modalities: plasticity and interactions. Curr Opin Neurobiol 11:505-509.

Simons DJ, Land PW (1987) Early experience of tactile stimulation influences organization of somatic sensory cortex. Nature 326:694-697.

Taddio A, Katz J, Ilersich AL, Koren G (1997) Effect of neonatal circumcision on pain response during subsequent routine vaccination. Lancet 349:599-603.

Visser GH, Laurini RN, de Vries JI, Bekedam DJ, Prechtl HF (1985) Abnormal motor behaviour in anencephalic fetuses. Early Hum Dev 12:173-182.

Weng HR, Schouenborg J (1998) On the cutaneous receptors contributing to withdrawal reflex pathways in the decerebrate spinal rat. Exp Brain Res 118:71-77.

Wenner P, O’Donovan MJ (2001) Mechanisms that initiate spontaneous network activity in the developing chick spinal cord. J Neurophysiol 86:1481-1498.

Woolf CJ (1996) Windup and central sensitization are not equivalent. Pain 66:105-108.

Woolf CJ, Wiesenfeld-Hallin Z (1985) The systemic administration of local anaesthetics produces a selective depression of C-afferent fibre evoked activity in the spinal cord. Pain 23:361-374.

Zhang LI, Poo MM (2001) Electrical activity and development of neural circuits. Nat Neurosci 4 [Suppl]:1207-1214. 